\title{
¿Es la ciencia abierta una respuesta válida para la lucha contra la desinformación científica?
}

\section{Is open science a valid answer to fight against scientific misinformation?}

\section{Alexandre López-Borrull}

López-Borrull, Alexandre (2021). "¿Es la ciencia abierta una respuesta válida para la lucha contra la desinformación científica?". Anuario ThinkEPI, v. 15, e15e06.

https://doi.org/10.3145/thinkepi.2021.e15e06

Publicado en IweTel el 16 de diciembre de 2021

\author{
Alexandre López-Borrull \\ https://orcid.org/0000-0003-1609-2088 \\ Universitat Oberta de Catalunya \\ Estudis de Ciències de la Informació i de la Comunicació \\ @alexandrelopez \\ alopezbo@uoc.edu
}

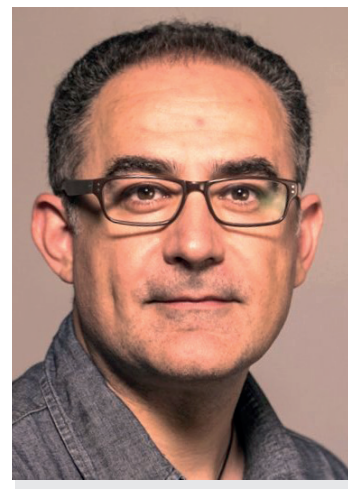

Resumen: La lucha contra la Covid-19 ha conllevado la necesidad de luchar contra la desinformación científica o fake science. La ciencia abierta como nuevo paradigma también tiene en cuenta la difusión del conocimiento científico hacia la sociedad. Se reflexiona sobre si la ciencia abierta puede ser una respuesta útil contra la desinformación y de qué manera se podría lograr.

Palabras clave: Ciencia abierta; Desinformación científica; Fake news; Fake science; Infodemia; Covid-19.

Abstract: The fight against Covid-19 has also led to the need to fight against scientific disinformation or fake science. Open science as a new paradigm also considers the dissemination of scientific knowledge towards society. This article reflects on whether open science can also be a useful response against misinformation and how this could be achieved.

Keywords: Open science; Misinformation; Disinformation; Fake news; Fake science; Infodemic; Covid-19.

\section{Introducción}

Anteriormente, en esta misma tribuna, hemos tenido la oportunidad de tratar la desinformación científica en relación con la Covid-19 y la gran cantidad de información (mucha de ella falsa), la llamada infodemia (López-Borrull, 2020). En esta nueva nota, quisiera reflexionar sobre si dos de los retos principales que según mi punto de vista tenemos como profesionales de la información, la ciencia abierta y la desinformación, tienen puntos en común, y cuáles serían las posibles sinergias que se pueden establecer en sus políticas y estrategias.

\section{Agradecimientos}

Este estudio se enmarca en el proyecto "Ciencia abierta en España: un enfoque global para evaluar su implementación" (RTI2018-094360-B-I00), financiado por el Plan Estatal I+D+i. 
Desde el pasado junio de 2021, Mercè Crosas es la nueva secretaria de Govern Obert (gobierno abierto) del Departament d'Acció Exterior i Transparència de la Generalitat de Catalunya. https://exteriors.gencat.cat/ca/detalls/noticia/not_210602_nomenament-MerceCrosas

Sin duda, tener a una de las coautoras de los principios FAIR (Wilkinson et al., 2016) es un valor añadido para cualquier administración. En especial, porque su visión de gobierno abierto que incluye a las universidades puede ayudar a cerrar la brecha que existe, según mi punto de vista, entre los datos abiertos de la administración y la visión de ciencia abierta en el mundo académico. Así, ya en la propia confluencia de caminos encontrados descrita por Fecher y Friesike (2014), el gobierno abierto y el concepto de open data no aparecen de forma clara, ni por su concepto ni por su filosofía. Es por ello por lo que los portales de transparencia y los repositorios en las propias universidades parecen tener vidas paralelas y demasiado separadas. Parece adecuado que alguien proveniente del campo académico tenga responsabilidades en la estrategia de compartición de datos públicos.

En una de sus primeras acciones, se organizaron unas jornadas anuales sobre gobierno abierto centradas en este caso en la Covid-19 con participación del colectivo académico. Tuve la oportunidad de poder intervenir en la segunda sesión titulada "Datos abiertos para frenar la curva"1. Algunas de las reflexiones asociadas a mi intervención me llevaron a reflexionar sobre si la ciencia abierta puede ser la contribución del mundo académico a la lucha contra la desinformación y sus derivadas. Apunto, pues, algunas ideas en relación con la pregunta del título para finalmente justificar mi respuesta positiva.

\section{2. ¿Qué hubiera pasado en la infodemia Covid-19 sin unos datos abiertos consensuados?}

Recientemente se han publicado una gran cantidad de artículos científicos centrados en la pandemia, la desinformación y las redes sociales, ya sea desde la óptica de los bulos (Salaverría et al., 2020); del discurso político (Pérez-Curiel; Velasco Molpeceres, 2020); los expertos científicos y la comunicación gubernamental (Elias, 2020) o la toxicidad de la política en Twitter (Guerrero-Solé; Philippe, 2020). Se aprecia así el interés por intentar entender y digerir un momento crítico y hacerlo desde la comprensión de los mecanismos de intercambio de información y comunicación. Teniendo pues en cuenta la polarización política presente por ejemplo en el Estado español (aunque también en Estados Unidos, Brasil y otros), debemos valorar positivamente cómo la validez de los datos proporcionados por la administración en relación a, por ejemplo, enfermos, hospitalizados y muertos por la enfermedad no se convirtieron en arma arrojadiza entre partidos políticos, aunque sí lo acabaran siendo las medidas de gestión, contención y prevención de la crisis, tanto legales como sanitarias.

Pero precisamente por la tensión política, cabe destacar, pues, que los datos que las distintas administraciones (jerárquicamente paralelas o concéntricas) fueran legitimados en el día a día. ¿Nos imaginamos qué hubiera sucedido si cada partido hubiera aparecido dando sus propios datos, o deslegitimando los datos de los demás antes de un confinamiento total o municipal? Y no será porque los políticos no sepan jugar con los datos para optimizar su mensaje político (Alonso-Pascual, 2021). Más allá del juego político y la campaña permanente, ¿cómo quedan estos datos ante la desinformación? Según mi punto de vista, la robustez y credibilidad de los datos abiertos fueron un muro infranqueable a menudo para las teorías negacionistas, allí donde chocaban los bulos de vídeos que intentaban demostrar que las ucis estaban vacías (Maldita.es, 2021), o bien que había un exceso de muertos no informados (Maldita.es, 2020). Asimismo, estos datos oficiales, públicos y en abierto hicieron también de cortafuegos para que determinados medios ofrecieran sus propias estimaciones creando realidades paralelas como actos de fe.

Sería oportuno, pues, reflexionar sobre el acierto. Sí, compartir datos en abierto parece haber sido muy útil para luchar contra la desinformación, por tanto, contra la infodemia y la pandemia. Sí, aunque seguro que ha habido errores en la compilación, agregación e interpretación, los datos compartidos también crearon un relato compartido ante el cual la desinformación "solo" pudo cuajar en aquellos que ya negaban la mayor, la existencia del propio virus o la conspiración de los chips.

Y esta misma idea es, según mi visión, escalable a la desinformación científica o fake science como se ha descrito anteriormente (López-Borrull; Ollé, 2019). Cuanto más abierta sea la ciencia, mejores mecanismos de verificación van a tener los ciudadanos o sus intermediadores, ya sean divulgadores científicos o bien periodistas especializados en ciencia. 


\section{3. ¿Es la ciencia abierta un paradigma catch-all?}

Si la memoria no me falla, hubo un momento en la implantación de internet en el cual, antes de la primera burbuja puntocom, un lugar común era el "no importa la pregunta, la respuesta es internet", en el cual se quería dejar claro que internet era la solución a todos los problemas preexistentes, y como tal, la sociedad y la ciudadanía debían adaptarse cuanto antes mejor. Esa visión optimista fue posteriormente modulada. ¿Puede pasarle lo mismo a la ciencia abierta? ¿No importa el problema, que la solución es la ciencia abierta? Diversos autores e informes han recogido reflexiones sobre la ciencia abierta y qué es como nuevo paradigma de la ciencia (Comisión Europea, 2016; Abadal; Anglada, 2020; Méndez, 2021).

Desde mi óptica, la complejidad de la implementación de la ciencia abierta y la dificultad de una hoja de ruta compartida y clara no son solo las posibles barreras o el cambio cultural, sino también que como sistema intenta resolver diversos problemas presentes en la ciencia y la sociedad a la vez:

- un ecosistema científico con grandes oligopolios privados y financiación mayoritariamente pública;

problemas con la reproducibilidad de la ciencia;

demasiado conocimiento creado con fondos públicos detrás de muros de pago;

dificultad de traslación a la sociedad del conocimiento y sus aplicaciones;

mecanismos de evaluación con sesgos y carencias;

un sector económico no suficientemente engrasado.

La visión europea de la ciencia abierta intenta corregir todos estos problemas con un paradigma que equilibra el balance ante ellos, aun a riesgo de añadir una mayor burocratización de la ciencia o generar críticas entre un colectivo científico que hace grandes sacrificios.

Aun así, en el momento inicial de la conceptualización y evolución de la ciencia 2.0 hacia la ciencia abierta de redacción de los informes y conceptos (Comisión europea, 2015), la desinformación no era citada ni era vista en aquel momento como algo nuclear ni emergente, ni cuando las fake news escalaron la comunicación política por culpa de Trump. Pero en estos momentos, después de una pandemia regada por desinformación masiva, parece oportuno plantear cómo la ciencia puede ayudar a luchar contra la desinformación. Así han tenido que hacerlo los medios de comunicación al principio dubitativos (lo que explicaría la aparición en este vacío de verificadores especializados como Maldita ciencia (Molina-Cabañate; Magallón-Rosa, 2020)), las bibliotecas en relación con si son garantes del conocimiento o la verdad (López-Borrull; Vives-Gràcia; Badell, 2018) o las plataformas y redes sociales con sus códigos de prácticas en desinformación.

https://digital-strategy.ec.europa.eu/en/policies/code-practice-disinformation

En este sentido, cuanto más sencilla y eficiente parece la viralización de contenidos falsos, incluso con mayor plausibilidad y calidad en el formato, mayor debe ser el paso de cada uno de los stakeholders en la lucha contra la desinformación y en favor de la verdad basada en evidencias. Los múltiples negacionismos (histórico, climático, sobre la violencia de género, etc.) así lo requieren.

\section{4. ¿Lo vivido durante la infodemia ha sido ciencia abierta?}

De la misma forma que el colega de universidad Albert Sangrà reflexionaba sobre que el paso drástico de la educación presencial a la virtual que tuvo lugar durante los confinamientos de la pandemia no podía considerarse e-learning como tal (López, 2020), también podemos preguntarnos si aquello que está teniendo lugar durante la pandemia está siendo ciencia abierta.

En primer lugar, la respuesta inicial sería que no, aunque es verdad que la propia Unesco citaba la ciencia abierta como un factor crítico en la lucha contra la Covid-19 (Unesco, 2020). Según mi punto de vista, hemos visto prácticas de ciencia abierta aceleradas de forma reactiva, respuestas legítimas y necesarias. Las principales editoriales y revistas dieron pasos ya en marzo de 2020 para la libre compartición de datos y artículos (Wellcome, 2020) y hubo un auge de preprints que aún está siendo estudiado (Torres-Salinas, 2020; Vlasschaert; Topf; Hiremath, 2020; Fraser et al., 2021). Dejo para el debate si los preprints en algunas disciplinas forman parte del problema o de la solución, puesto que se vislumbran como mal menor respecto al enorme retraso en la publicación de muchas revistas académicas. Algo similar ocurrió con los datos de investigación, aunque algunos artículos recientes ponen en duda la eficacia de algunas de estas llamadas a los inves-

"Cuanto más sencilla y eficiente parece la viralización de contenidos falsos, incluso con mayor plausibilidad y calidad en el formato, mayor debe ser el paso de cada uno de los stakeholders en la lucha contra la tigadores (Lucas-Domínguez et al., 2021). 
Pero la ciencia abierta debe ayudar a la mejora del sistema, crear un ecosistema diferente donde las bases estén hechas para que el conocimiento fluya más libre, desde su diseño a su creación, desde las políticas de financiación a las de evaluación. Y siempre con la calidad como bandera, como el propio Plan S por ejemplo deja claro. Es cierto que cada vez más políticas científicas recogen algunos de los aspectos de la ciencia abierta como el acceso abierto a las publicaciones o bien la gestión y difusión de los datos de investigación, pero aún con una cierta falta de protocolos claros y un balance entre el palo y la zanahoria a medio camino entre la excepción y la generalización.

No se intuya aquí una crítica a las prácticas de ciencia abierta llevadas a cabo por el colectivo científico. Al contrario, tiene mucho mérito que, con un andamio precario, con muchas dudas, incertidumbres y sacrificios hayan difundido como nunca conocimiento. Pero no se utilicen los artículos retirados por temas metodológicos o falsos datasets como un ataque a la ciencia abierta, sino como malas prácticas de algunos científicos que en algún caso llegaron a tener un alto impacto mediático debido a la necesidad acuciante de noticias, sobre todo positivas (López-Borrull, 2020). Por tanto, se debe sin duda apostar por el camino que se ha abierto, no creer que todo fue una ilusión pandémica, ni que ya no haya más que hacer o que la ciencia abierta aparecerá por generación espontánea.

\section{5. ¿Lanzarse "sin red" contra la desinformación es una opción contra los científicos?}

Asimismo, hay que recordar que uno de los componentes de la ciencia abierta pasa también por la comunicación de conocimiento científico y difusión a la sociedad, cosa que en estos momentos pasa por los medios de comunicación, pero también por las redes sociales, ya sean académicas o generales. En este sentido, se publicó en octubre un artículo en Nature escrito por Nogrady (2021) donde se hacía eco de una encuesta realizada a personal científico que había expresado y compartido contenidos en las redes en relación con la Covid-19 durante la pandemia. La encuesta hacía aflorar una problemática, dado que

"más de dos tercios de los investigadores respondieron que habían tenido experiencias negativas como resultado de sus apariciones en los medios de comunicación o sus comentarios en las redes sociales, y el $22 \%$ habían recibido amenazas de violencia física o sexual" (Nogrady, 2021).

Sin duda, pero no por primera vez, difundir conocimiento en las redes sociales o tener presencia en los medios de comunicación ha tenido un coste para los científicos. Pero habrá que reconocer el esfuerzo. Hay que recordar también en este sentido cómo ya en la propia visión del proyecto Foster (2021) aparecían las redes sociales como lugar para la difusión de conocimiento científico (figura 1). De nuevo, será básico relacionar ciencia abierta con calidad. Porque en el caso de la desinformación científica y como apuntaba recientemente Elisabeth Bik, la ganadora del premio John Maddox,

"el peligro con las redes sociales es que incluso un artículo mediocre, malo o defectuoso puede ser tomado por personas que tienen diferentes agendas y ser objeto de atención y celebrarse como la nueva verdad" (Devlin, 2021).

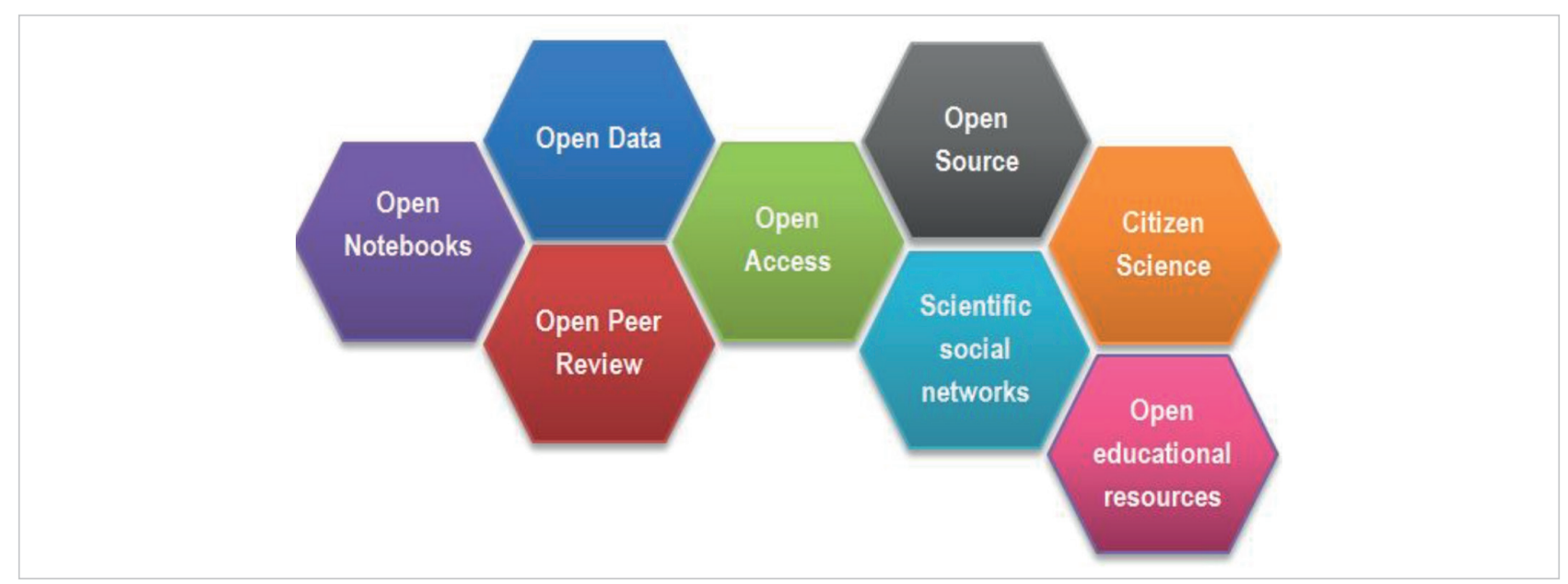

Figura 1. Las facetas de la ciencia abierta como una colmena.

Fuente: $h t t p s: / / w w w$.fosteropenscience.eu/content/what-open-science-introduction 


\section{Conclusión: sí, una ciencia abierta y de calidad contra la desinformación científica}

Sí, la ciencia abierta puede ser también una respuesta en la lucha contra la desinformación. Es más, en el camino hacia la implementación debe hacerse énfasis en el concepto de ciencia abierta y de calidad. Así, debe servirle también para apartarse y diferenciarse de las prácticas depredadoras con las que únicamente comparte el acceso abierto a los contenidos. Y es en la calidad y la difusión de conocimiento científico hacia la sociedad donde va a poder aportar contenido de valor que pueda refutar la desinformación, ser capaz de llegar directamente a la ciudadanía y a los medios de comunicación. Se apuntan algunas ideas y recomendaciones que podría ayudar en este sentido:

- Las políticas de ciencia abierta deberán hacer más énfasis en la difusión del conocimiento científico también hacia la sociedad, tomando la idea de la Investigación y la Innovación Responsable (RRI, por sus siglas en inglés) de la ciencia "con y para la sociedad" y asumir que una óptima diseminación científica en abierto es también una herramienta en la lucha contra la desinformación.

- Las políticas de lucha contra la desinformación, sobre todo en relación con el cambio climático y la Covid-19, deben considerar la ciencia abierta como expresión natural de un espacio compartido entre el colectivo científico y el resto de la sociedad. Así, además de los medios de comunicación y las plataformas y redes sociales, deberían incorporar en sus grupos de trabajo representantes de instituciones académicas y bibliotecarias.

- Científicos, pero también instituciones y bibliotecas universitarias, deben proveer de contenidos de calidad de los avances científicos que se están produciendo, así como participar de los debates (y hashtags) en las redes sociales, aun a riesgo de la exposición mediática y de odio que puede tener lugar. Sin duda, es mayor la ayuda a la mayoría silenciosa que el minoritario pero ruidoso espacio negacionista.

- Entender la difusión de conocimiento y la divulgación científica en medios y redes sociales como transferencia y que ello sea apremiado por las instituciones, a la vez que valorado y reconocido.

En los próximos años, la ciencia debe ser también central en relación con el cambio climático y la llamada a la acción de emergencia publicada paralelamente en más de 200 publicaciones académicas ${ }^{2}$ es una buena muestra de este papel central que la ciencia debe tener. Aunque a veces ello implique situarse en la diana del odio presente en las redes sociales, habrá que valorar las ventajas de dicha presencia y entender que el reconocimiento social del colectivo científico es un valor a la hora de analizar la información y el conocimiento que se difunde en las redes en el momento actual, con una crisis de las democracias liberales que cuestiona la clase política, la justicia y el papel de los propios medios de comunicación. Y la ciencia abierta puede ser, también, la palanca de cambio para una mejor sociedad. Ojalá.

"La ciencia abierta y de calidad
es una respuesta eficaz contra
la desinformación y debe incluir
instituciones, bibliotecas y el colectivo
científico"

\section{Notas}

1. Dades obertes per a frenar la corba. https://www.youtube.com/watch?v=mKQ5f92LmcM\&t=6400s

2. Optamos por citar un medio que se hizo eco de la publicación ante la dificultad de elegir una revista concreta https://www.lavanguardia.com/natural/20210906/7701822/mas-200-revistas-cientificas-reclaman-acciones-urgentescambio-climatico.html

\section{Referencias}

Abadal, Ernest; Anglada, Lluís (2020). "Ciencia abierta: cómo han evolucionado la denominación y el concepto". Anales de documentación, v. 23, n. 1.

https://doi.org/10.6018/analesdoc.378171

Alonso-Pascual, Cristina (2021). "Los cinco trucos que usan los políticos para manipular los datos de empleo a su favor". Newtral, 9 septiembre.

https://www.newtral.es/datos-empleo-politicos-manipulacion/20210909/

Comisión Europea (2015). Validation of the results of the public consultation on science 2.0: Science in transition. https://www.eesc.europa.eu/resources/docs/validation-of-the-results-of-the-public-consultation-on-science-20.pdf

Comisión Europea (2016). Open innovation. Open science. Open to the world. A vision for Europe. https://op.europa.eu/en/publication-detail/-/publication/3213b335-1cbc-11e6-ba9a-01aa75ed71a1 
Devlin, Hannah (2021). "Misinformation fuelled by 'tsunami' of poor research, says science prize winner". The guardian, 1 December.

https://www.theguardian.com/science/2021/dec/01/misinformation-fuelled-by-tsunami-of-poor-research-saysscience-prize-winner

Elías, Carlos (2020). “Expertos/as científicos/as y comunicación gubernamental en la era de las fake news: Análisis de la estrategia informativa del Covid-19 en España". Prisma social, n. 31, pp. 6-39.

https://revistaprismasocial.es/article/view/3945

Fecher, Benedikt; Friesike, Sascha (2014). "Open science: One term, five schools of thought". En: Bartling, Sönke; Friesike, Sascha (eds.) Opening Science. Springer Open, pp. 17-47.

https://doi.org/10.1007/978-3-319-00026-8_2

Foster (2021). The future of science is open.

https://www.fosteropenscience.eu

Fraser, Nicholas; Brierley, Liam; Dey, Gautam; Polka, Jessica K.; Pálfy, Mate; Nanni, Federico; Coates, Jonathon-Alexis (2021). "The evolving role of preprints in the dissemination of Covid-19 research and their impact on the science communication landscape". PLoS biology, v. 19, n. 4, e3000959.

https://doi.org/10.1371/journal.pbio.3000959

Guerrero-Solé, Frederic; Philippe, Olivier (2020). “La toxicidad de la política española en Twitter durante la pandemia de la Covid-19". Hipertext.net, n. 21, pp. 133-139.

https://doi.org/10.31009/hipertext.net.2020.i21.12

López, Agustín (2020). “El aprendizaje en línea en 2021: de la urgencia a la calidad". UOC news, 15 diciembre. https://www.uoc.edu/portal/es/news/actualitat/2020/463-elearning-2021-calidad.html

López-Borrull, Alexandre (2020). "Fake news e infodemia científica durante la Covid-19, ¿dos caras de la misma crisis informacional?". Anuario ThinkEPI, v. 14, e14e07.

https://doi.org/10.3145/thinkepi.2020.e14e07

López-Borrull, Alexandre; Ollé, Candela (2019). "La curación de contenidos científicos como respuesta a las noticias y a la ciencia falsas". Anuario ThinkEPI, v. 13, e13e07.

https://doi.org/10.3145/thinkepi.2019.e13e07

López-Borrull, Alexandre; Vives-Gràcia, Josep; Badell, Joan-Isidre (2018). "Fake news, ¿amenaza u oportunidad para los profesionales de la información y la documentación?". El profesional de la información, v. 27, n. 6, pp. 1346-1356.

https://doi.org/10.3145/epi.2018.nov.17

Lucas-Dominguez, Rut; Alonso-Arroyo, Adolfo; Vidal-Infer, Antonio; Aleixandre-Benavent, Rafael (2021).

"The sharing of research data facing the Covid-19 pandemic". Scientometrics, n. 126, pp. 4975-4990.

https://doi.org/10.1007/s11192-021-03971-6

Maldita.es (2021). "Por qué no debes fiarte de los vídeos que muestran zonas de hospitales vacíos y afirman que la Covid es mentira". Maldito bulo, 1 febrero.

https://maldita.es/malditobulo/20210201/videos-hospitales-vacios-coronavirus

Maldita.es (2020). "No, el Gobierno no oculta estas imágenes de féretros como dice Vox: fueron emitidas por TVE y grabadas por la agencia EFE". Maldito bulo, 4 abril.

https://maldita.es/malditobulo/20200404/no-el-gobierno-no-oculta-estas-imagenes-de-feretros-como-dice-voxfueron-emitidas-por-tve-y-grabadas-por-la-agencia-efel

Méndez, Eva (2021). “Open science por defecto. La nueva normalidad para la investigación”. Arbor, v. 197, n. 799, a587.

https://doi.org/10.3989/arbor.2021.799002

Molina-Cañabate, Juan-Pedro; Magallón-Rosa, Raúl (2020). “Desinformación y periodismo científico. El caso de Maldita Ciencia". Revista mediterránea de comunicación, v. 11, n. 2, pp. 11-21.

https://www.doi.org/10.14198/MEDCOM2020.11.2.4

Nogrady, Bianca (2021). "'I hope you die': how the Covid pandemic unleashed attacks on scientists". Nature, n. 598, pp. 250-253.

https://doi.org/10.1038/d41586-021-02741-x

Pérez-Curiel, Concha; Velasco-Molpeceres, Ana-María (2020). "Impacto del discurso político en la difusión de bulos sobre Covid-19. Influencia de la desinformación en públicos y medios". Revista latina de comunicación social, n. 78, pp. 65-97.

https://doi.org/10.4185/RLCS-2020-1469 
Salaverría, Ramón; Buslón, Nataly; López-Pan, Fernando; León, Bienvenido; López-Goñi, Ignacio; Erviti, Maria-Carmen (2020). "Desinformación en tiempos de pandemia: tipología de los bulos sobre la Covid-19". Profesional de la información, v. 29, n. 3.

https://doi.org/10.3145/epi.2020.may.15

Torres-Salinas, Daniel (2020). "Ritmo de crecimiento diario de la producción científica sobre Covid-19. Análisis en bases de datos y repositorios en acceso abierto". Profesional de la información, v 29, n. 2.

https://doi.org/10.3145/epi.2020.mar.15

Vlasschaert, Caitlyn; Topf, Joel M.; Hiremath, Swapnil (2020). “Proliferation of papers and preprints during the Coronavirus disease 2019 pandemic: Progress or problems with peer review?". Advances in chronic kidney disease, v. 27 , n. 5, pp. $418-426$.

https://doi.org/10.1053/j.ackd.2020.08.003

Wellcome (2020). "Publishers make coronavirus (COVID-19) content freely available and reusable". Wellcome, 16 March.

https://wellcome.org/press-release/publishers-make-coronavirus-covid-19-content-freely-available-and-reusable

Wilkinson, Mark D.; Dumontier, Michel; Aalbersberg, ljsbrand J.; Appleton, Gabrielle; Axton, Myles; Baak, Arie; Blomberg, Niklas; Boiten, Jan-Willem; Bonino-da-Silva-Santos, Luiz; Bourne, Philip E.; Bouwman, Jildau; Brookes, Anthony J.; Clark, Tim; Crosas, Mercè; Dillo, Ingrid; Dumon, Olivier; Edmunds, Scott; EveIo, Chris T.; Finkers, Richard; González-Beltrán, Alejandra; Gray, Alasdair J. G.; Groth, Paul; Goble, Carole; Grethe, Jeffrey S.; Heringa, Jaap; Hoen, Peter A.C 't; Hooft, Rob; Kuhn, Tobias; Kok, Ruben; Kok, Joost; Lusher, Scott J.; Martone, Maryann E.; Mons, Albert; Packer, Abel L.; Persson, Bengt; Rocca-Serra, Philippe; Roos, Marco; Van-Schaik, Rene; Sansone, Susanna-Assunta; Schultes, Erik; Sengstag, Thierry; Slater, Ted; Strawn, George; Swertz, Morris A.; Thompson, Mark; Van-der-Lei, Johan; Van-Mulligen, Erik; Velterop, Jan; Waagmeester, Andra; Wittenburg, Peter; Wolstencroft, Katherine; Zhao, Jun; Mons, Barend (2016). "The FAIR guiding principles for scientific data management and stewardship". Sci Data, n. 3, 160018.

https://doi.org/10.1038/sdata.2016.18

Unesco (2020). Open access to facilitate research and information on Covid-19.

https://en.unesco.org/covid19/communicationinformationresponse/opensolutions

\section{Inforảrea}

Ayudamos a tu organización en la transformación digital yel gobierno de la información

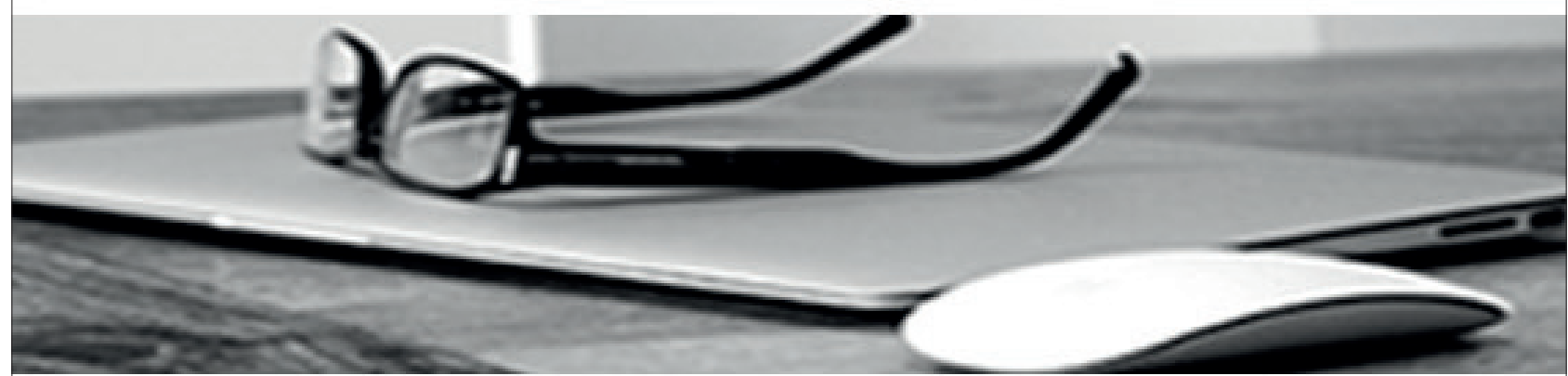

* Consultoría estratégica en gestión y gobierno de la información

* Gestión documental y "records management"

* Gestión de contenidos, intranets corporativas y entornos de colaboración

* Estudios especializados

Clientes satisfechos, cientos de empresas nacionales e internacionales y más de 30 años de experiencia son la mejor garantía de nuestra reputación. 